\title{
SNPs in ERCC1 and drug response to cisplatin in non-small-cell lung cancer patients
}

"SNPS ... have been known to be predictive markers of treatment response, toxicity, time to disease progression and survival in cancer patients."

\section{KEYWORDS: cisplatin $\approx E R C C 1 \backsim \mathrm{SNP}$}

Pharmacogenomics can be a useful tool to predict drug responsiveness (e.g., toxicity) as well as outcome, including survival. It can therefore also be used to engineer individual treatment according to the genetic characteristics of cancer patients. The target for phamacogenomics is genetic information on polymorphisms and gene expression. The major approach involves the SNPs (a point mutation) which have been known to be predictive markers of treatment response, toxicity, time to disease progression and survival in cancer patients.

There are several general methods for the genotyping of SNPs [1]. One such method is PCRRFLP. This lacks powerful high throughput, which means that it cannot be used for the identification of all genotypes. Quantitative reverse transcription (RT)-PCR is limited by its cost, whereas the 5'-nuclease assay for allelic discrimination is relatively new. Another recent method, 3D polyacrylamide gel-based DNA microarray, has been shown to be inexpensive and extremely powerful for the genotyping of many samples.

“...cisplatin is most widely known as a scaffolding of combination chemotherapy in NSCLC."

Lung cancer is one of the leading common causes of cancer-related deaths worldwide. Non-small-cell lung cancer (NSCLC) constitutes $>85 \%$ of cases of primary lung cancer and approximately two thirds of patients with NSCLC are diagnosed at an advanced stage [2]. Moreover, NSCLC is a chemoresistant tumor. The prognosis for patients with NSCLC is dismal regardless of the chemotherapy regimen used [3].

The standard care for patients with advanced NSCLC is platinum-based chemotherapy. Platinum is one of the most extensively used chemotherapeutic agents and remains the firstline treatment in various types of cancer [4].
Three commonly used platinum compounds are cisplatin, carboplatin and oxaliplatin. Crosslinks between guanine bases are induced by cisplatin, carboplatin and oxaliplatin. Cisplatin and carboplatin form an identical crosslink, whereas the oxaliplatin crosslink is structurally very different in the adduct [5]. The formation of platinum-DNA adducts that result in DNA crosslinks inhibit transcription, block replication and lead to the death of cancer cells. Hence, survival is expected to be longer in patients with higher levels of platinum-DNA adducts. Of the platinum compounds, cisplatin is most widely known as a scaffolding of combination chemotherapy in NSCLC. The combination of cisplatin with a third-generation cytotoxic drug (e.g., vinorelbine, gemcitabine, paclitaxel or docetaxel) is the standard chemotherapy for first-line treatment of advanced-stage (stage IIIB or IV) NSCLC [6]. The International Adjuvant Lung Cancer Trial (IALT) found a modest benefit of $4.1 \%$ in 5-year overall survival among 1867 patients treated with adjuvant cisplatin-based chemotherapy [7]. However, drug resistance and the detrimental effects of chemotherapy may reduce the quality of life of patients. Therefore, a plateau has been reached in the efficacy of chemotherapy.

\section{“...the repair/removal of DNA adducts has been demonstrated to be the 'trigger' that leads to cisplatin resistance because NER-deficient cells are hypersensitive to cisplatin."}

DNA adducts of cisplatin are mainly removed by the nucleotide excision repair (NER) pathway. This is one of the four DNA repair mechanisms that has a role in the control of the progression of the cell cycle and apoptosis via DNA damage checkpoints. The NER pathway can be divided into two distinct subpathways. These are called

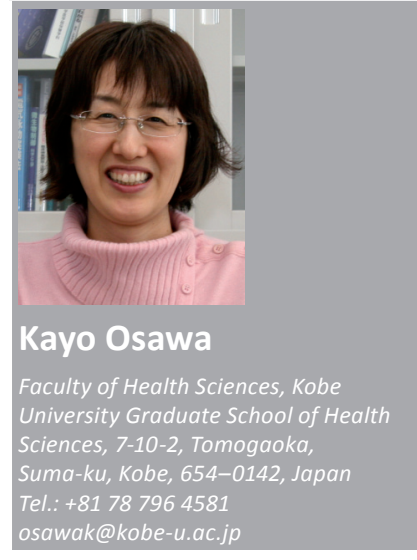

future medictio $^{\text {pisg }}$ fs 
the transcription-coupled repair (TCR) pathway (which repairs transcription-blocking lesions in transcribed DNA strands of active genes) and the global genomic repair pathway (which repairs the lesions in the nontranscribed strand of active genes and the nontranscribing genome) $[3,8]$. In the TCR pathway, damage is detected by the elongating RNA polymerase II complex when it encounters a lesion [9]. Thus, the tumors are sensitive to cisplatin if the TCR pathway is deficient [8]. Therefore, the repair/removal of DNA adducts has been demonstrated to be the 'trigger' that leads to cisplatin resistance because NERdeficient cells are hypersensitive to cisplatin. The reduced capacity to repair DNA may be expected to lead to an increased response and prolongation of survival for the cisplatin-based chemotherapy.

\section{“...pharmacogenetic studies for the SNPS of ERCC1 may be used as predictive and prognostic markers of outcome for NSCLC patients treated by cisplatin-based chemotherapy."}

Of the proteins involved in the excision of damaged base(s) in the TCR pathway, excision repair cross-complementation group 1 (ERCC1) is a rate-limiting enzyme $[1,10]$, which forms a heterodimer with xeroderma pigmentosum $\mathrm{F}$ (XPF) [3]. The ERCC1/XPF complex is responsible for the incision that cleaves the damaged strand at the 5'-end of the DNA lesion, particularly those induced by interstrand crosslinks. Therefore, ERCC1 is important for the repair of platinum-DNA adducts. The IALT-Bio program recently demonstrated that patients with ERCC1-negative (low-expression) tumors derived a substantial benefit from adjuvant cisplatin-based chemotherapy compared with ERCC1-positive (high-expression) tumors [11]. Other studies observed a decreased risk of death in patients with ERCC1-negative tumors as compared with patients with ERCC1-positive tumors after cisplatin-based adjuvant chemotherapy [2]. ERCC1 overexpression is also associated with resistance to cisplatin-based chemotherapy in NSCLC patients [12]. The capacity for DNA repair on the basis of low or high expression of ERCC1 can be a determinant of the sensitivity to cisplatin-based chemotherapy in NSCLC patients.

Furthermore, the two common SNPs of ERCC1; one at codon $118 \mathrm{C} / \mathrm{T}$ (which is associated with differential mRNA levels and protein levels) and C8092A (which is located in the 3'-UTR of the gene and which may affect ERCC1 mRNA stability) have been recognized [13,14]. The SNP at codon 118 of ERCC1 can influence the resistance/sensitivity of platinum [15]. Studies demonstrated that ERCC1 codon $118 \mathrm{C} / \mathrm{T}$ was correlated with time-todisease progression and treatment response, as well as survival in cisplatin-based chemotherapy in NSCLC patients $[1,16-19]$. Contrary to expectation, it has been reported that patients with wild-type $E R C C 1 \mathrm{C} / \mathrm{C}$ had a significantly longer survival and time to progression than those harboring non-wild-type ERCC1 [19]. As to the reason, there was no significant difference between ERCC1 protein expression levels and the ERCC1 SNP. With respect to function, the increase in protein expression in codon 118 of ERCC1 has also been associated with diminished survival [16].The C8092A SNP has been associated with overall survival, response and outcome in patients with advanced NSCLC treated by cisplatin-based chemotherapy [1,20]. Other studies demonstrated no significant association between outcome, treatment response and overall survival in the two SNPs of ERCC1 [4,21]. Therefore, pharmacogenetic studies for the SNPs of ERCC1 may be used as predictive and prognostic markers of outcome for NSCLC patients treated by cisplatin-based chemotherapy.

\section{Financial \& competing interests disclosure}

The author has no relevant affiliations or financial involvement with any organization or entity with a financial interest in or financial conflict with the subject matter or materials discussed in the manuscript. This includes employment, consultancies, honoraria, stock ownership or options, expert testimony, grants or patents received or pending, or royalties.

No writing assistance was utilized in the production of this manuscript.

\section{Bibliography}

1 Li F, Sun X, Sun N et al.: Association between polymorphisms of ERCC1 and XPD and clinical response to platinum-based chemotherapy in advanced non-small cell lung cancer. Am. J. Clin. Oncol. 33, 489-494 (2010).
2 Chen S, Zhang J, Wang R et al.: The platinum-based treatments for advanced non-small cell lung cancer, is low/negative ERCC1 expression better than high/positive ERCC1 expression? A meta-analysis. Lung Cancer 70, 63-70 (2010).

3 Rosell R, Taron M, Alberola V et al.: Genetic testing for chemotherapy in non-small cell lung cancer. Lung Cancer 41, S97-S102 (2003).

4 Yin M, Yan J, Voutsina A et al.: No evidence of an association of ERCC1 and ERCC2 polymorphisms with clinical outcomes of platinum-based chemotherapies in non-small cell lung cancer: a meta-analysis. Lung Cancer (2010) (Epub ahead of print). 
5 Siddik ZH: Mechanisms of action of cancer chemotherapeutic agents: DNA-interactive alkylating agents and antitumour platinumbased drugs. In: The Cancer Handbook. Alison MR (Ed.). Nature Publishing Group, London, UK, 1295-1313 (2002).

6 Viñolas N, Provencio M, Reguart N et al:: Single nucleotide polymorphisms in MDR1 gen correlates with outcome in advanced non-small-cell lung cancer patients treated with cisplatin plus vinorelbine. Lung Cancer 71, 191-198 (2011).

7 Arriagada R, Bergman B, Dunant A et al.; The International Adjuvant Lung Cancer Trial Collaborative Group: Cisplatin-based adjuvant chemotherapy in patients with completely resected non-small-cell lung cancer. N. Engl. J. Med. 350, 351-360 (2004).

8 Furuta T, Ueda T, Aune G et al.: Transcriptioncoupled nucleotide excision repair as a determinant of cisplatin sensitivity of human cells. Cancer Res. 62, 4899-4902 (2002).

9 de Laat WL, Jaspers NG, Hoeijmakers JH: Molecular mechanism of nucleotide excision repair. Genes Dev. 13, 768-785 (1999).

10 de Laat WL, Appeldoorn E, Jaspers NG, Hoeijmakers JH: DNA structural elements required for ERCC1-XPF endonuclease activity. J. Biol. Chem. 273, 7835-7842 (1998).
11 Olaussen KA, Dunant A, Fouret P et al: : DNA repair by ERCC1 in non-small-cell lung cancer and cisplatin-based adjuvant chemotherapy. N. Engl. J. Med. 355, 983-991(2006).

12 Kartalou M, Essigmann JM: Mechanisms of resistance to cisplatin. Mutat. Res. 478, 23-43 (2001).

$13 \mathrm{Yu} J \mathrm{~J}, \mathrm{Mu} \mathrm{C}$, Lee $\mathrm{KB}$ et al.: A nucleotide polymorphism in ERCC1 in human ovarian cancer cell lines and tumor tissues. Mutat. Res. 382, 13-20 (1997).

14 Chen P, Wiencke J, Aldape K et al.: Association of an ERCC1 polymorphism with adult-onset glioma. Cancer Epidemiol. Biomarkers Prev. 9, 843-847 (2000).

15 Smith S, Su D, Rigault de la Longrais IA et al.: ERCC1 genotype and phenotype in epithelial ovarian cancer identify patients likely to benefit from paclitaxel treatment in addition to platinum-based therapy. J. Clin. Oncol. 25, 5172-5179 (2007).

16 Zhou C, Ren S, Zhou $S$ et al:: Predictive effects of ERCC1 and XRCC3 SNP on efficacy of platinum-based chemotherapy in advanced NSCLC patients. Jpn. J. Clin. Oncol. 40, 954-960 (2010).
17 Kalikaki A, Kanaki M, Vassalou H et al.: DNA repair gene polymorphisms predict favorable clinical outcome in advanced non-small-cell lung cancer. Clin. Lung Cancer 10, 118-123 (2009).

18

Wei SZ, Zhan P, Shi MQ et al.: Predictive value of ERCCI and XPD polymorphism in patients with advanced non-small cell lung cancer receiving platinum-based chemotherapy: a systematic review and meta-analysis. Med. Oncol. 28(1), 315-321 (2011).

19 Isla D, Sarries C, Rosell R et al.: Single nucleotide polymorphisms and outcome in docetaxel-cisplatin-treated advanced non-small-cell lung cancer. Ann. Oncol. 15, 1194-1203 (2004).

20 Takenaka T, Yano T, Kiyohara C et al.: Effects of excision repair crosscomplementation group 1 (ERCC1) single nucleotide polymorphisms on the prognosis of non-small cell lung cancer patients. Lung Cancer 67, 101-107 (2010).

21 Tibaldi C, Giovannetti E, Vasile E et al.: Correlation of $C D A, E R C C 1$, and XPD polymorphisms with response and survival in gemcitabine/cisplatin-treated advanced non-small cell lung cancer patients. Clin. Cancer Res. 14, 1797-1803 (2008). 